\title{
$X X$. The effect of the presence of hydrogen on the intensity of the lines of the carbon spectrum
}

\section{Arthur M. Herbert B.A.}

To cite this article: Arthur M. Herbert B.A. (1902) XX. The effect of the presence of hydrogen on the intensity of the lines of the carbon spectrum , Philosophical Magazine Series 6, 4:20, 202-207, DOI: $10.1080 / 14786440209462834$

To link to this article: http://dx.doi.org/10.1080/14786440209462834

曲 Published online: 15 Apr 2009.

Submit your article to this journal $[\pi$

Џll Article views: 3

Q View related articles $\square$ 
place at different times of the day and under different atmospheric conditions, and thus escaping the notice of even an expert observer, Rayleigh's formula regarding the composition of the diffuse light of the sky is not verified numerieally, it does not follow that Rayleigh's theory is not to be retained as furnishing the best explanation of the colour of the atmosphere. Variations in the number and size of the particles which give rise to the turbidity of the atmosphere, and in what is still more irregular-equally invisible strata of water-vapour in the lower regions-explain sufficiently, in accordance with Rayleigh's theory, the discrepancies among the complex results which hare been obtained in the strata just above the surface of the soil-strata forming 'atmospherie mud.'

"At the present time, the controversial choice lies between the colour proper of the air and Rayleigh's theory. This theory has an energetic and staunch supporter in Prof. Pernter, who, as we have seen, has several times replied to Spring, combating the latter's views. In the present state of science, Rayleigh's theory is the only one capable of explaining satisfactorily certain observed facts of special importance in connexion with the colour of the atmosphere, of which the principal one is that at an angle of $90^{\circ}$ we get the maximum of polarization by the atmosphere, and of light reflected by a turbid medium-a fact which, according to Pernter, 'is the experimentum crucis with regard to the theory of the blue colour of the sky.'

"I shall conclude by quoting Pernter:-

" "The turbid medium called air is that which gives rise to the blue colour of the sky. The weak colour proper of the air-if it exists-contributes nothing towards it."

XX. The Effect of the Presence of Hydrogen on the Intensity of the Lines of the Carbon Spectrum. By ARTHUR M. Herbert, B.A.*

TN a paper "On the Spectra of some of the Chemical Elements," in the Philosophical Transactions for 1864, Sir William Huggins remarks that "when carbon is subjected to the induction-spark in the presence of hydrogen the strong line in the red (a little less refrangible than the hydrogen red line corresponding to Fraunhofer's $\mathrm{C}$ in the solar spectrum) is not seen." This peculiarity is referred to by him once again in counexion with certain cometary spectra

* Communicated by Prof. Arthur Schuster, F.R.S. 
the Intensity of the Lines of the Carbon Spectrum. 203

in a later paper (Phil. Trans. 1868), so that apparently be considered the result to be unquestioned.

As no details of the experiments were given, and in fact no further reference to this particular question could be found anywhere, the following experiments were undertaken at Professor Schuster's suggestion, whose constant advice I had the advantage of in the conduct of them.

The red line of carbon is a double one $\left(\lambda_{6577}^{6583}\right)$, the more refrangible one being the stronger. When the slightest trace of hydrogen was present along with the carbonic acid gas, in which the spark was taken in the first instance, the red line of hydrogen ( $\lambda$ 6562) appeared; as more and more hydrogen was admitted it was found that the carbon red line very rapidly diminished in brightness, so that with a comparatively small proportion of hydrogen in the mixture the carbon line appeared very faint.

The other principal line of carbon seen when the spark is taken in carbonic acid is a broad one in the violet $(\lambda+266)$. The behaviour of this line in the presence of hydrogen was quite different; as the relative proportion of hydrogen was increased this line merely suffered a gradual diminution in brightness (along with several oxygen lines in its neighbourhood), but it never disappeared, being quite distinct when the amount of hydrogen present was far in excess of the carbonic acid; indeed it was quite recognizable with an exceedingly small proportion of carbonic acid in the mixture, and seemed, if anything, to be more persistent than the oxygen lines near it in the violet.

Two other carbon lines were examined $(\lambda 5640$ and $\lambda 5145)$; they apparently suffered merely a gradual weakening as the proportion of hydrogen was increased, but as they are much fainter than the two principal lines they were not attended to in the subsequent experiments, which were directed solely to investigating the difference in behaviour of the two strong lines. T'hus it appeared from these initial experiments that the red line of carbon seen under these conditions is affected by the presence of hydrogen in a totally different way from the other carbon lines.

After these few preliminary experiments, in which Sir William Huggins's observations were generally confirmed, several series of experiments were made with mixtures of carbonic acid and hydrogen in known proportions by volume. The gases, supplied by two Kipp's apparatus, were passed into an aspirator which was graduated so that definite volumes of each gas could be admitted; from the aspirator 


\section{Mr. A. M. Herbert on the Effect of Hydrogen on}

the gas, or mixed gases, was forced through the dischargetube, after bubbling through strong sulphuric acid to get rid of most of the moisture present. A slow current of gas was maintained so as to prevent the accumulation in the discharge-tube of any products of chemical decomposition due to the passage of the spark.

The spark passed between platinum-iridium electrodes which were about $3 \mathrm{~mm}$. apart; these were fused into the bulb of the discharge-tube, the diameter of the bulb being about $4 \mathrm{~cm}$. One of Apps's induction-coils was used which gave a spark of about 3 inches between its terminals in air, when four Tudor cells ( 2 volts each) supplied the primary current.

A small leyden-jar, the outer tinfoil area being about $280 \mathrm{sq} . \mathrm{cm}$., was included in the circuit. In all the experiments the spark was taken at atmospheric pressure.

The following results were obtained, each set of experiments being repeated several times with similar results; the effects upon the two red lines are noted :-

A. Spark in mixture of Carbonic Acid Gas and Hydrogen.

(i.) $2 \frac{1}{2}$ parts $H$ in 25 parts mixture $=10$ per cent. $H$. [Hydrogen line stronger than

(ii.) 5

(iii.) 5

(iv.) 7

(v.) 1
25

25

17

14

20
$=20$ per cent. H. [ $=30$ per cent. $\mathrm{H}$. (approx.) $=50$ per cent. $\mathrm{H}$.

$=5$ per cent. $\mathrm{H}$. carbon line.]

Hydrogen line strong, carbon line faint.]

[Hydrogen line very strong, carbón line very faint.]

[Carbon line hardly distinguishable.]

[Lines about equally strong; hydrogen, if anything, slightly stronger.]

A second set of experiments gave the following results :-

(i.) $2 \frac{1}{2}$ parts $\mathrm{H}$ in 25 parts mixture $=10$ per cent. $\mathrm{H}$. [Hydrogen line slightly the

(ii.)

(iii.) 10

(iv.) $12 \frac{1}{2}$

(v.) $\frac{1}{2}$

(vi.) 1
20

25

25

50

20 stronger.]

$=30$ per cent. $H$. [Hydrogen line very strong, arbon line very faint.]

$=40$ per cent. H. [Oarbon line hardly distinguishable.]

$=50$ per cent. H. [Carbon line hardly visible.]

$=1$ per cent. $H$. [Carbon line very strong; hydrogen line very faint, but quite distinct.]

$=5$ per cent. $\mathrm{H}$. carbon, if anything, slightly stronger.]

The percentages given do not, of course, pretend to any great accuracy; thus there did not seem to be much difference in the effects between 40 per cent. and 50 per cent. of hydrogen. 
It will be noticed that in the first set of experiments the hydrogen line appeared slightly the stronger with כ per cent. hydrogen in the mixture, whereas in the second set the carbon line was slightly the stronger with the same percentage. This is probably due to the fact that the vessel containing sulphuric acid through which the gas bubbled was, after each experiment, left full of the mixture just used, so that this contaminated, especially at first, the next mixture. In the first case 5 per cent. hydrogen was tried immediately after 50 per cent. hydrogen, so that probably the hydrogen was somewhat in excess of 5 per cent. during most of the time. In the second case the preceding mixture contained mostly carbonic acid, so that in this case the hydrogen would probably be slightly less than 5 per cent. in the mixture during most of the time. It is likely, therefore, that with 5 per cent. hydrogen in the mixture the two lines are about equally strong.

As before stated, the violet strong line of carbon is not affected to this extent; it was examined in each experiment, and it merely suffered a natural weakening as the proportion of carbonic acid diminished, but it was quite distinct with 90 per cent. of hydrogen in the mixture, and easily visible with still more bydrogen.

Before proceeding any further the effect of nitrogen (or rather, air) was tried; this simply weakened both carbon lines more or less equally ; with 80 per cent. of air, for example, both carbon lines were quite distinct and fairly bright.

The experiments were now repeated, using higher dispersion so as to separate the two red lines still further. Two prisms of dense glass were used, and a small screen was fixed in the eyepiece, which could be moved along horizontally by means of a screw-head so as to cover up the hydrogen line, and hence to eliminate all possibility of optical illusion due to contrast effects. The results were substantially the same as before; it appeared that with 50 per cent. of hydrogen the carbon red line is still faintly visible but not readily distinguishable-it seemed to flash in and out faintly rather than to be faintly persistent. At times I thought it was present, at other times I was sure it was not, so that its presence seemed to depend upon very unstable conditions.

B. Spark in mixture of Carbon Monoxide and Hydrogen.

(i.) 19 parts $\mathbf{H}$ in 38 parts mixture $=50$ per cent. $\mathrm{H}$. [Hydrogen line very strong;

(ii.) $1 \quad$ " $20 \quad, \quad=5$ per cont. H. [Lines about equal.]

(iii.) $9 \quad " \quad 32 \quad " \quad=28$ per cent. H. [Hydrogen line strong, car-

(iv.) $22 \quad, \quad 44 \quad, \quad=50$ per cent, H. [Carbon line hardly distinguishable.] 
206 Effect of Ifydrogen on the Lines of the Carbon Spectrum.

Several other series of experiments with mixtures of carbonic oxide and hydrogen were made, and the results agreed together, and with the corresponding results obtained with mixtures of carbonic acid and hydrogen so far as conld be estimated by the conditions of the experiments.

\section{Spark in Coal-gas.}

The spark in coal-gas showed the red hydrogen line strongly--the other hydrogen lines were not attended tothe violet carbon line well, but the red carbon line was hardly visible. Taken as a whole, the appearance of these three lines was much the same as in a mixture of carbonic acid and hydrogen containing 50 per cent. of hydrogen.

The effect of mixing coal-gas with carbonic acid was then tried with the following results :-

(i.) 5 per cent. coal-gas in the mixture.

(ii.) 10

(iii.) 33

$\begin{array}{ll}", & , \\ , & ,\end{array}$

[Carbon red line slightly the stronger.] [Hydrogen line slightly the stronger.]

[Carbon red line faint, but quite distinguisibable.]

(iv.) 50

[Carbon line very faint, but. more readily seen than with 50 per cent. hydrogen.]

It will be seen that the red line of carbon is more persistent than in the case of corresponding mixtures of carbonic acid and hydrogen; this is only to be expected, as the coalgas brings carbon as well as hydrogen into the mixture.

From these experiments the conclusion seems certain that though the red carbon line may not be completely destroyed by the presence of hydrogen-at any rate when the proportion of hydrogen does not exceed 50 per cent. - yet it is influenced in a way totally different from the other strong line of carbon in the violet, whereby it suffers a very rapid quenching, as the proportion of hydrogen is increased, to which the violet line is not subject.

It might, perhaps, be thought that the effects observed are due to the faint carbon line being rendered invisible owing to the strong glare of the hydrogen red line in its neighbourhood. But this possible objection is, I think, completely met by the consideration of the following facts.

Firstly, the two lines were well separated by the instrument used, and the screen fixed in the eyepiece enabled either one of them to be covered up; secondly, when a mixture containing a mere trace of hydrogen was examined the very faint persistent hydrogen line was seen without any difficulty by the side of the very bright carbon line; and, lastly, when, with 50 per cent. hydrogen, the carbon red line appeared to flash 
in and out, it was quite distinctly seen when it flashed in, and its flashing out was equally distinct.

It has been suggested to me by Professor Schuster that the experiments seem to show that the red and violet carbon lines must belong to different spectra of carbon, the particular molecular combination which gives rise to the red line being destroyed by the presence of hydrogen. The fact that the two interfering lines lie near each other in the spectrum is probably accidental. I have to thank Professor Schuster also for many other suggestions made during the course of the experiments.

XXI. The Laws of Electrolysis of Alkali Salt-Vapour's. By Harold A. Wilson, D.Sc., M.Sc., B.A., Clerk-Maxwell Student, Fellow of Trinity College, Cambridge*.

TN 1891 Arrhenius (Wied. Ann. xlii. p. 18, 1891) published the results of an investigation on the passage of electricity through flames containing salt-vapours, and proposed the theory that the salts dissociate into ions in the flame in the same way that salts are ionized in aqueous solutions.

Arrhenius' results were confirmed and extended in 1899 in a research initiated by Prof. A. Smithells and carried out in conjunction with Dr. H. M. Dawson and the writer (Phil. Trans. A. 1899). Since then the writer has published (Phil. Trans. A. 1899 and 1901) the results of further work which seem to show conclusively that conduction through salt-vapours is accomplished by means of ions of some lind, and is therefore to this extent at least analogous to conduction through solutions.

The experiments now to be described were undertaken with the object of determining the relative conductivities of different alkali salt-vapours at various temperatures. Many of the results obtained have been published in a paper on the "Electrical Conductivity of Air and of Salt-Vapours," read to the Royal Society this year.

In aqueous solutions a salt such as $\mathrm{KCl}$ dissociates into two ions $+\mathrm{K}$ and $-\mathrm{Cl}$, so that the most likely supposition is that in salt-vapours the ions are of the same nature.

However, determinations of the velocities of the various ions in salt-vapours show that the ions generally behave as if they were much heavier than single atoms, and that the positive ion always moves more slowly than the negative ion.

* Communicated by the Author. (A paper read to the Britrsh Association, Glasgow, 1901.) 